\title{
Late Abstracts
}

P-P-25

Fluoride concentration in bottled water, soft drinks
and fruit beverages
D. DAVIS-WALLACE*, A. MENENDEZ, L. ROJAS,
J. JACKSON \& J. RUBY
Department of Pediatric Dentistry, The University of
Alabama at Birmingham, Birmingham, AL, USA

Dental fluorosis is a chronic condition caused by excess fluoride intake during tooth development that results in disrupted enamel formation and hypomineralization. Excess fluoride intake from birth to age three will result in white spots (mild) or brown discolourations (severe) on the labial surfaces of the permanent central incisors which may present aesthetic concerns to the child and the parents. These highly visible cosmetic defects are not trivial, for they are noticeable when speaking or smiling, potentially engendering behavioural selfconsciousness. Fluorosis has doubled in suboptimally fluoridated (<0.7 p.p.m. fluoride) areas in the United States from the $1930 \mathrm{~s}(6.5 \%)$ to the $1980 \mathrm{~s}(15.5 \%)$. This increase in fluorosis has been attributed to the accumulative intake of fluoride from a variety of sources including toothpaste, supplemental fluorides and infant formula. We present evidence that bottled water, soft drinks and fruit beverages contain fluoride that may also contribute to accumulative fluoride intake, thereby increasing the risk of fluorosis in this vulnerable age group (birth to age three). Bottled water $(n=17)$, soft drinks $(n=7)$ and fruit beverages $(n=11)$ were purchased from a variety of grocery and convenience stores in the Birmingham, Alabama area. In addition, water samples $(n=2)$ were collected from the same area's municipal water supply at different times from distal outlets The fluoride analysis was performed by OMNII Pharmaceuticals using duel Orion electrodes and the fluoride ion concentration was recorded as parts per million or milligrams per litre. All bottled water, soft drinks and fruit beverages contained fluoride. The range of fluoride ion concentration among the samples tested in each group, was: bottled water (range $=0.03-0.83$ p.p.m.), soft drinks (range $=0.21-0.35$ p.p.m.), fruit beverages (range $=0.09-$ 0.78 p.p.m.). Children in nonfluoridated areas could be drinking significant levels of bottled water, soft drinks and fruit beverages containing fluoride, thereby enhancing their accumulative fluoride intake.

*Presenting author.
DM-P-24

Vitapex pulpectomy for infected primary teeth H. KO*, F. RODRIGUEZ, M. BAJJALIEH \& J. RUBY Department of Pediatric Dentistry, The University of Alabama at Birmingham, Birmingham, AL, USA

Pulpectomy in primary teeth is indicated treatment for inflamed or necrotic pulps, and as alternative therapy for vital pulpotomy treatment. The usual treatment protocol is: the pulp chamber is accessed, the pulpal contents within the chamber and canals are extirpated with files and irrigated, the canals are then dried with absorbent points and filled with a root canal sealer such as zinc oxide eugenol (ZOE). Several problems may arise when performing this procedure: 1) Primary molar root canals are tortuous and difficult to access or instrument. 2) ZOE placement is problematic, e.g. broken spiral 'lentulos' lodged in canals, extrusion of sealer beyond the root apex and inadequate sealing of the tortuous molar root canal system. Moreover, ZOE does not resorb well, extruded ZOE sealer beyond the root apex may function as a foreign body and ZOE sealer within the canals may interfere with the normal eruption of the underlying succedaneous tooth; both requiring surgical removal of residual ZOE. Recently, Vitapex (iodoform $40.4 \%$, calcium hydroxide $30.3 \%$, silicone oil $22.4 \%$ ) (DiaDent, Burnaby, British Columbia, Canada) has been introduced as a root canal dressing and sealer for pulpectomy in primary teeth by Carlos Nurko and Franklin Garcia-Godoy. Vitapex has several advantageous properties: 1) iodoform and calcium hydroxide are effective antiseptics for eliminating any remaining infection. 2) Extruded calcium hydroxide beyond the apex resorbs. 3) Silicone oil provides optimal flow characteristics for proper sealing. 4) Ease of application remixed in a deliverable syringe with disposable tip. This case series presents follow-up examinations and radiographs of six Vitapex pulpectomy treatments in infected primary teeth. All of the cases presented had successful therapeutic outcomes.

DM-P-23

Prevalence and correlates of traumatic dental injuries in Taiwan

B. HUANG*, R. CROUCHER, M. HECTOR \& W. MARCENES

Queen Mary's School of Medicine and Dentistry, Queen Mary, University of London, London, UK

Objectives. To assess the prevalence of traumatic dental injuries (TDI) in Taiwan, and to analyse the relationships between the occurrence of traumatic dental injuries and 
school type, age, gender, socio-economic status, family structure, overjet and lip morphology.

Methods. A random sample of 6284 15-18-year-old academic and vocational senior high school students in southern Taiwan was selected. Each completed a questionnaire and was examined with standard clinical procedures.

Results. Participation rate was $99.97 \%$. The prevalence of TDI in Taiwan was $19.94 \%$ (95\% C.I. $18.96 \%, 20.95 \%)$. Male adolescents $(\mathrm{OR}=1.809,95 \%$ C.I. 1.589, 2.058) attending private $(\mathrm{OR}=1.158,95 \%$ C.I. $1.004,1.336)$ and/or vocational schools $(\mathrm{OR}=1.200,95 \%$ C.I. $1.030,1.398)$, with an overjet greater than $5 \mathrm{~mm}(\mathrm{OR}=1.697,95 \%$ C.I. $1.450,1.985)$, inadequate lip morphology $(\mathrm{OR}=1.350,95 \%$ C.I. $1.189,1.533)$, living with at least one nonbirth parent $(\mathrm{OR}=1.274,95 \%$ C.I. $1.013,1.601)$, and/or having the middle educated chief guardian $(\mathrm{OR}=1.210,95 \%$ C.I. $1.039,1.411)$, were found to be at greater risk of TDI.

Conclusions. The prevalence of TDI in Taiwan was similar to that found in other countries. Correlates of TDI were gender, lip morphology, overjet, chief guardian's level of education, family structure and school type. Further investigation into the aetiology of TDI is indicated. 